\title{
Relationship between Deceleration Areas in the Second Stage of Labor and Neonatal Acidemia
}

\author{
Angela Agostinelli, Flavio Palmieri, Alessandra Biagini, Agnese Sbrollini, Luca Burattini, \\ Francesco Di Nardo, Sandro Fioretti, Laura Burattini* \\ Department of Information Engineering, Università Politecnica delle Marche, Ancona, Italy
}

\begin{abstract}
Understanding the fetal heart rate (FHR) in the second stage of labor is important to recognize critical clinical outcomes. During this time, fetus is subject to head compression that activate baroreceptor reflexes that cause FHR deceleration. Large deceleration areas (DA: measured as duration by depth), have been associated to critical fetal outcomes. A preliminary study on 33 pregnant women reported a significant inverse correlation between DA in the last 60 minutes before delivery and umbilical $\mathrm{pH}$ at birth (fetal-distress measure when $\leq$ 7.1). The aim of this study is to further characterize the relationship between DA in the last 60 minutes before delivery and $\mathrm{pH}$ at birth on a larger population. Thus, 433 FHR recordings from the "CTUCHB Intrapartum Cardiotocography Database" of Physionet were used. Signals were classified as Cases $(p H \leq 7.1)$ and Controls $(p H>7.1)$. Results confirmed that Cases have significantly higher DA than Controls $\left(5.32 \mathrm{~cm}^{2}\right.$ vs $\left.1.44 \mathrm{~cm}^{2} ; P<0.05\right)$. The inverse correlation between DA in the last 60 minutes before delivery and $p H$ at birth, was weak but significant $\left(\rho=-0.23, P<10^{-6}\right)$. Thus, results confirm that critical fetal outcomes increase with increasing acidemia; however, such relationship may not be strictly linear. Future studies are focused on discrimination of fetal distress using DA.
\end{abstract}

\section{Introduction}

Cardiotocography (CTG), also known as electronic fetal heart-rate monitoring, was introduced in 1958 assess fetal well-being during labor [1]. CTG performs auscultation and counting of fetal heart-rate (FHR) during a uterine contraction (UC) [2]. Indeed, as shown in Fig. 1, it consists of two simultaneous recordings (FHR and UC signals) accomplished by means of two separate sensors: a Doppler ultrasound sensor for FHR detection and a pressure transducer for UC detection. Since transducers can be internal or external, CTG is usually performed in noninvasive fashion by using a hand-held probe or external transducer placed on the maternal abdomen by an elastic belt [2].

FHR is controlled by the sympathovagal balance of the fetal nervous system [3]. In the fetal hypoxia condition, sympathovagal balance is altered causing continuous adjustments of FHR in order for it to stay in the normal range (110-160 bpm) [4]. Since fetal hypoxia during labor, especially in the second stage of labor, may cause fetal distress [5], understanding FHR variations in the second stage of labor is of great importance to recognize some critical clinical outcomes [6]. The second stage of labor, which begins when the cervix is full dilated and ends with delivery, has a duration usually ranged between 60 minutes (in nulliparas) and 30 minutes (for multiparas) [7]. During this time, the fetus is often subject to reduced oxygenation [3]. Particularly, UC (which may reach up to $100 \mathrm{mmHg}$ pressure to make fetal head to descend through the pelvis) causes a fetal head compression [6] that causes an increasing of endocranic pressure and a reduction of cerebral flow. Those changes activate baroreceptor reflexes that cause a severe FHR deceleration [5]. If this deceleration is synchronous to UC, it is called "early deceleration"; otherwise, it is called "late deceleration" [8]. While the first is generally considered as a physiological reaction, the second represents a pathologic condition and the fetus may not well tolerate both entity and length of deceleration [2]. Indeed, values of the deceleration area (DA), measured as deceleration duration by depth, have been found to be associated to critical fetal outcomes [9]. In 2013, a preliminary study [10] attempted to quantify this phenomenon previously only qualitatively described. The study, accomplished on 33 pregnant women with FHR 


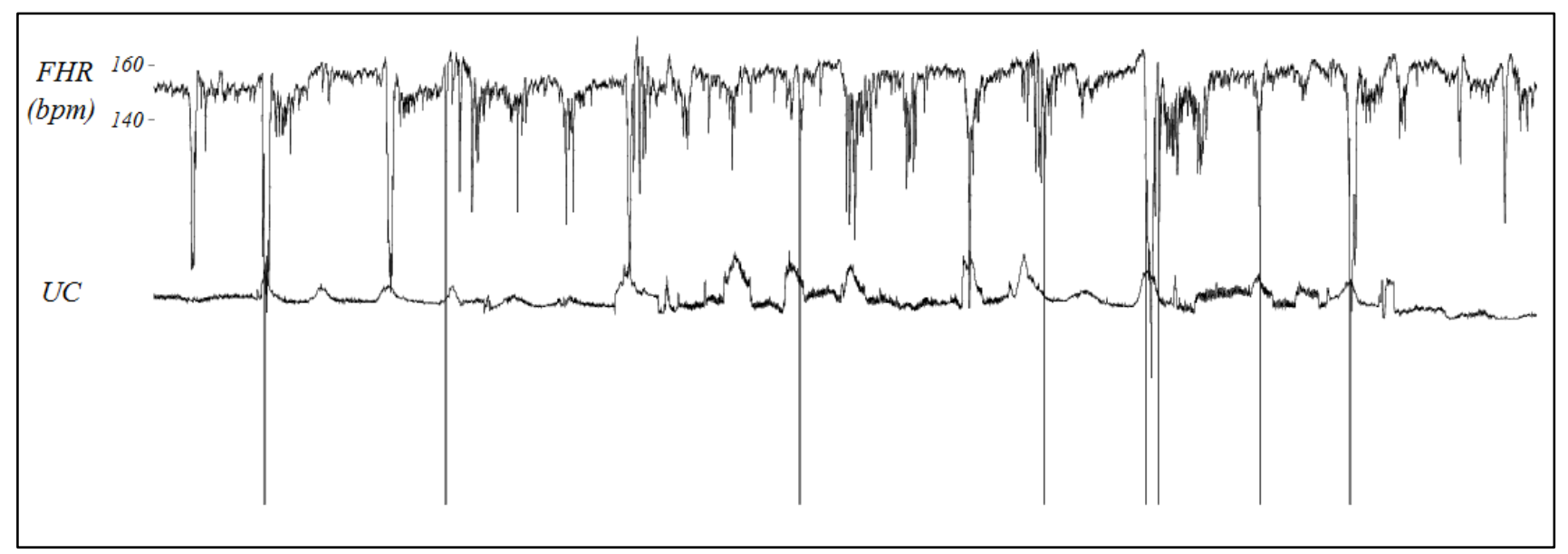

Fig. 1. Example of CTG recording (record 1020) available at “CTU-CHB Intrapartum Cardiotocography Database” of Physionet). Upper panel: the fetal heart-rate time series (FHR, bpm), lower panel: uterine contraction signal (UC).

decelerations in the second stage of labor, reported a significant inverse correlation between DA measured in the last 60 minutes before delivery and umbilical $\mathrm{pH}$ at birth, which is considered a measure of fetal distress when $\leq 7.1$. Aim of the present work was to further characterize the relationship between DA measured in the last 60 minutes before the delivery and $\mathrm{pH}$ at birth on a larger population.

\section{Clinical data and methods}

Clinical data consisted of 552 CTG recordings containing FHR and a UC signals both sampled at $4 \mathrm{~Hz}$ and constituting the "CTU-CHB Intrapartum Cardiotocography Database” [11] of Physionet [12] (http://physionet.org/physiobank/database/ctu-uhb-ctgdb). CTG recordings were acquired by the Czech Technical University in Prague and the University Hospital in Brno. They start no more than 90 minutes before actual delivery and each is at most 90 minutes long. The database is characterized by the following criteria:

- $\quad$ singleton pregnancies;

- gestational age > 36 weeks;

- no a priori knowledge of developmental defects;

- $\quad$ second stage of labor not longer than 30 minutes;

- FHR signal quality (i.e. percentage of the recording during which FHR data were available) $>50 \%$ in each 30 minute window;

- availability of biochemical parameters of umbilical arterial blood sample;
- majority of vaginal deliveries (only 46 cesarean deliveries included).

- availability of neonatal $\mathrm{pH}$ at birth, obtained from the analysis of the umbilical artery blood sample.

In order to have a homogeneous data set, this study considered only CTG acquisitions for which the last hour of FHR recording (which thus also included delivery) was characterized by at least $90 \%$ of valid data (not available data were replaced by mean value). Only these 60-min FHR windows were analyzed in the present study.

As in [10], DA calculated for FHR $\leq 90$ bpm was correlated with umbilical $\mathrm{pH}$ at birth that was used to classify FHR tracings as Cases, when umbilical neonatal $\mathrm{pH} \leq 7.1$, and Controls, when umbilical neonatal $\mathrm{pH}>$ 7.1 .

DA distributions relative to Cases and Controls, were described in terms 50 (median) [25;75] percentiles and compared using the Wilcoxon Rank-Sum Test. Association between DA values and neonatal $\mathrm{pH}$ was evaluated by computing of the Pearson's correlation coefficient $(\rho)$ and the area (AUC) under the receiver operating characteristic (ROC). Statistical significance level was set at 0.05 for all tests.

\section{Results}

Out of the 552 FHR recordings available in the database, $433(80 \%)$ satisfied the inclusion criteria and were thus analyzed in the present study. Of these, 34 were classified as Cases and 399 as Controls. Median DA values for Cases was 5.32 [0;34.29] $\mathrm{cm}^{2}$ and median DA values for Controls was $1.44[0 ; 13.62] \mathrm{cm}^{2}$. Globally, 


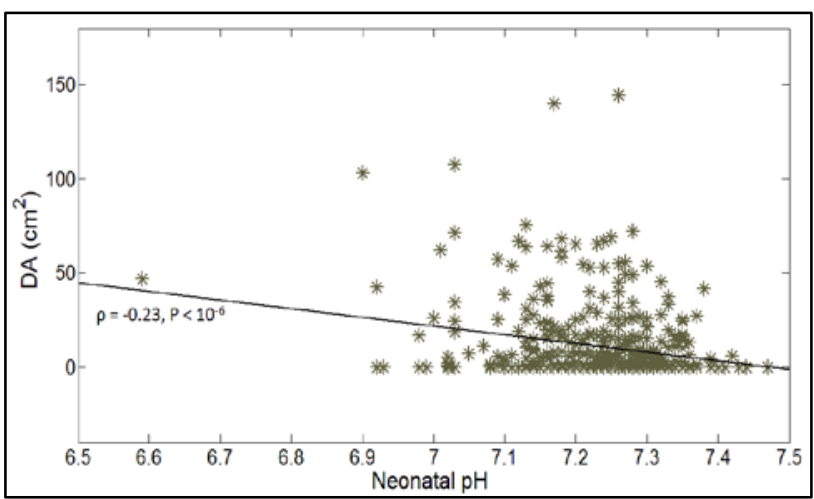

Fig. 2. Deceleration areas (DA) in the last 60 minutes before delivery as function of $\mathrm{pH}$ at birth with relative regression line.

results confirmed that Cases have significantly higher DA than Controls (5.32 vs $1.44, \mathrm{P}<0.05)$. A weak although significant inverse correlation $\left(\rho=-0.23, \mathrm{P}<10^{-6}\right)$ was observed between DA in the last 60 minutes before delivery and $\mathrm{pH}$ at birth (Fig. 2). ROC relative to $\mathrm{pH}$ at birth is reported in Fig. 3; AUC was found to be 0.61 .

\section{Discussion}

CTG represents the most commonly used technique for fetal monitoring in clinics, and is a technological application to predict and reduce neonatal critical outcomes [13]. Although CTG is highly reliable for fetal hypoxia identification [14], its predicting value could be enhanced by combining it with fetal electrocardiography (FECG) which records the electrical activity of the fetal heart [15]. Indeed, beside FHR evaluation, FECG allows to have additional information on fetal well-being based on FECG signal morphology, such as ST-segment [1620]. However, FECG may increase risk of infection when the electrode is directly placed on the fetal scalp [21] or may require significant signal-processing to reduce noise when the electrode in placed on the mother's abdomen [22,23].

Previous studies have suggested a link between fetal hypoxia during labor, especially in the second stage of labor, and fetal distress $[5,10]$. In turn, fetal hypoxia cause FHR decelerations, each characterized by a duration and a depth. Consequently, DA can be used to characterize a deceleration. Instead, fetal distress can be quantitatively characterized by measuring the $\mathrm{pH}$ at birth. Thus, this study followed what done in [10] on a population of 33 pregnant women and attempted a characterization of the relationship between DA measured in the last 60 minutes before delivery and $\mathrm{pH}$ at birth on a much larger population. In order to have a homogeneous

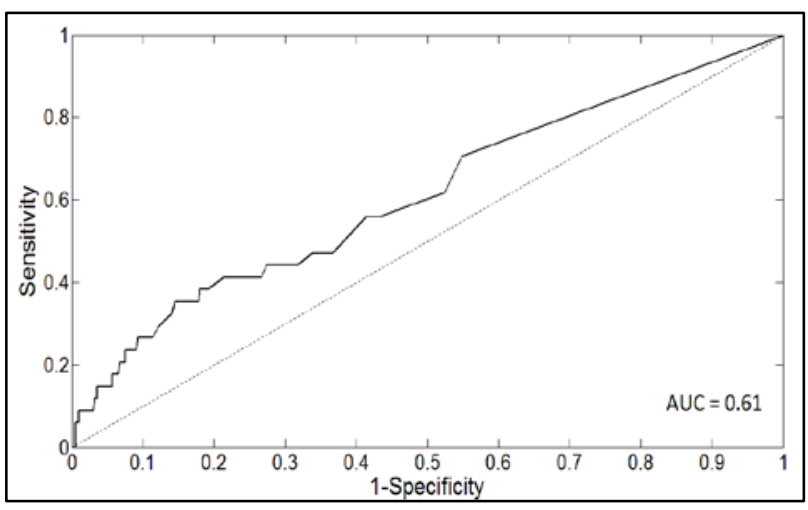

Fig. 3. Receiver operating characteristic (ROC, solid line) with the relative area under curve (AUC) relative to $\mathrm{pH}$ at birth.

data set that maintains the same study conditions of [10], 433 60-min FHR windows before delivery were selected from of the 552 available at the "CTU - CHB Intrapartum Cardiotocography Database" of Physionet. To be selected, 60-min FHR windows had to have at least 90\% available data. Since FHR could present any spikes (as can be shown in Fig. 1) to $0 \mathrm{bpm}$ related to losses of the contact skin by the sensor, these spikes were corrected in order to enhance the signal quality. In addition, in order to correlate DA and umbilical $\mathrm{pH}$ at birth, FHR tracings were classified as Cases (umbilical neonatal $\mathrm{pH} \leq 7.1$ ) and Controls (umbilical neonatal $\mathrm{pH}$ > 7.1).

According to our results, DA for Cases were always significantly higher than Controls $\left(5.32 \mathrm{~cm}^{2}\right.$ vs $1.44 \mathrm{~cm}^{2}$; $\mathrm{P}<0.05$ ), thus confirming was observed in [10]. In addition DA was inversely correlated to $\mathrm{pH}$ at birth, as observed in [10], but correlation was much lower $(\rho=-$ $0.23, \mathrm{P}<10^{-6}$ ) than what previously observed $(\rho=-0.77$; $\mathrm{P}<0.05$ [10]). The fact that fetal bradycardia (in terms of $\mathrm{DA})$ in the 60 minutes preceding delivery is significantly correlated with the decreasing of neonatal $\mathrm{pH}$, confirms that critical fetal outcomes increase with increasing neonatal acidemia; however, such relationship may not be strictly linear (as the hypothesized by the correlation coefficient). A possible cause of discrepancy between the correlation values found here and in [10] may be due to the presence of several records in which FHR signal has not DA (as it can be seen in Fig. 2). The same not happens in [10], where all FHR recordings in the study population presented severe bradycardia below $90 \mathrm{bpm}$ in the second stage of labor. In addition, the different dimension of study populations (which is equal to 433 for this study and only 33 for the preliminary study) can affect the correlation value.

ROC curve confirms that neonatal $\mathrm{pH}$ is characterized by a quite low discriminating power. Indeed, AUC was found to be 0.61 (whereas should be $\geq 0.8$ to have a high 
accuracy value). Given the results found here, DA represents a possible discrimination parameter for identification of fetuses with distress.

\section{Conclusion}

DA represents a severe fetal bradycardia; associates to critical fetal outcomes and increases with increasing neonatal acidemia. Further investigations are needed to discriminate the fetal distress cases using DA.

\section{References}

[1] Alfirevic Z, Devane D, Gyte GML. Continuous cardiotocography (CTG) as a form of electronic fetal monitoring (EFM) for fetal assessment during labour. Cochrane Db Syst Rev 2013, Issue 5, pp. 1-136.

[2] Sweha A, Hacker TW. Interpretation of the Electronic Fetal Heart Rate During Labor. Am Fam Physician 1999;59:2487-2500.

[3] Kamath MV, Fallen EL. Power spectral analysis of heart rate variability: a noninvasive signature of cardiac autonomic function. Crit Rev Biomed Eng 1993;21:245311.

[4] Heart rate variability. Standards of measurement, physiological interpretation, and clinical use. Task Force of the European Society of Cardiology and the North American Society of Pacing and Electrophysiology. Eur Heart J 1996;17:354-81.

[5] Talaulikar VS, Lowe V, Arulkumaran S. Intrapartum fetal Surveillance. Obstet Gynaecol Reprod Med 2014;24:4555.

[6] Biagini A, Landi B Tranquilli AL. The clinical significance of bradycardia in the second stage of labor. Giornale Italiano di Ostetricia e Ginecologia CIC Edizioni Internazionali 2013; 35(6): 717-21.

[7] Cunningham FG, Gant NF, Leveno KJ, et al. Conduct of normal labor and delivery. In: Williams obstetrics $21^{\text {st }}$ ed. New York, USA: Mc Graw-Hill, 2001:105-831.

[8] Hon EH. The electronic evaluation of the fetal heart rate. Am J Obstet Gynecol 1958;75:1215-30.

[9] Beguin F, Yeh SY, Forsythe A, et al. A study of fetal heart rate deceleration areas. II. Correlation between deceleration areas and fetal $\mathrm{pH}$ during labor. Obstet Gynecol. 1975;45:292-8

[10] Tranquilli AL, Biagini A, Greco P, et al. The Correlation Between The Area Of Fetal Bradycardia In The Second Stage Of Labor And Acidemia At Birth. J Matern Fetal Neonatal Med 2013;26:1425-9.

[11] Chudáček V, Spilka J, Burša $M$, et al. Open access intrapartum CTG database. BMC Pregnancy and Childbirth 2014;14-6.

[12] Goldberger AL, Amaral LAN, Glass L, et al. PhysioBank, PhysioToolkit, and PhysioNet: Components of a New Research Resource for Complex Physiologic Signals. Circulation 2000;101:e215-20.

[13] Feinstein NF, Sprague A, Trepanier MJ. Comparing Auscultation to Electronic Fetal Monitoring. AWHONN Lifelines 2000;4:35-44.
[14] Tasnim N, Mahmud G, Akram S. Predictive accuracy of intrapartum cardiotocography in terms of fetal acid base status at birth. J Coll Physicians Surg Pak. 2009;19:632-5.

[15] Agostinelli A, Grillo M, Biagini A, et al. Noninvasive Fetal Electrocardiography: An Overview of the Signal Electrophysiological Meaning, Recording Procedures, and Processing Techniques. Ann Noninvasive Electrocardiol 2015;20:303-13.

[16] Baschat AA. Integrated fetal testing in growth restriction: Combining multivessel Doppler and biophysical parameters. Ultrasound Obst Gyn 2003;21:1-8.

[17] Hornberger LK, Sahn DJ. Rhythm abnormalities of the fetus. Heart 2007;93:1294-1300.

[18] Kleinman CS, Nehgme RA. Cardiac arrhythmias in the human fetus. Pediatr Cardiol 2004;25:234-51.

[19] Neilson JP. Fetal electrocardiogram (ECG) for fetal monitoring during labour. Cochrane Database Syst Rev 2013;5:1-29.

[20] Sadovsky E, Polishuk WZ. Fetal movements in utero nature, assessment, prognostic value, timing of delivery. Obstet Ginecol 1977;50:49-55.

[21] Westerhuis MEMH, Visser GHA, Moons KGM, et al. Cardiotocography Plus ST Analysis of Fetal Electrocardiogram Compared With Cardiotocography Only for Intrapartum Monitoring. A Randomized Controlled Trial. Obstet Gynecol 2010;115:1173-80.

[16] Agostinelli A, Giuliani C, Burattini L. Extracting a clean ECG from a noisy recording: a new method based on segmented-beat modulation. Computing in Cardiology 2014; 41:49-52.

[17] Agostinelli A, Sbrollini A, Giuliani C et al. Segmented beat modulation method for electrocardiogram estimation from noisy recordings. Med EngPhys 2016; 38(6): 560-8.

Address for correspondence.

Laura Burattini

Department of Information Engineering

Università Politecnica delle Marche

Via Brecce Bianche, 60131 Ancona, Italy

l.burattini@univpm.it. 